\title{
Salt leaching due to rain in Mediterranean climate: is it enough?
}

\author{
Massimo Monteleone, Angela Libutti \\ Dipartimento di Scienze Agro-Ambientali, Chimica e Difesa Vegetale, Università di Foggia, Italy
}

\begin{abstract}
The increasing limitation of available water resources for agriculture raises the issue of an appropriate use of low quality water (particularly brackish or saline water) for agricultural productivity without jeopardizing the quality of soil and its productive capacity. Referring to typical Mediterranean climate conditions and assuming a systematic irrigation use of brackish groundwater, this paper analyzes the capability of yearly rainfall, particularly in fall-winter period, to leach the salts accumulated in the soil during the previous spring-summer irrigation season. The leaching capability of water supplies exceeding the soil water holding capacity has undergone direct evaluation through a particular experimental arrangement: under a rain shelter, soil columns (inside special cylindrical containers), previously salinized and bare at the surface, were treated with repeated irrigations. Fresh water was used for this purpose, in order to simulate rainwater. The amounts and proportions of salt removed from the soil as well as the relative quantity of salt left in the soil were monitored. An appropriate statistical data analysis led to the interpretation of the observed process by developing a leaching curve able to predict the fraction of salts remaining along the soil profile according to the height of leaching water added to the soil, expressed as a fraction of the depth of the soil layer considered. According to the experimentally determined leaching curve (related to a silt-loam textured soil, basically unstructured and compacted as a result of a prolonged salinization), the following rule of thumb can be taken: the application of a defined height of leaching fresh water reduces by $70 \%$ (i.e. reduces to $30 \%$ ) the salt content of a soil layer of equal depth. The elaboration of this conve-
\end{abstract}

Correspondence: Massimo Monteleone, Dipartimento di Scienze AgroAmbientali, Chimica e Difesa Vegetale, Università di Foggia, via Napoli 25, 71122 Foggia, Italy. Tel. +39.0881.589223. E-mail: m.monteleone@unifg.it

Key words: brackish water, drainage water, leaching curve, salts leaching, soil salinity.

Acknowledgements: this research was funded by CLIMESCO Evolution of cropping systems as affected by climate change project, contract n. 285, 20/02/2006 (Ministry for Education, University and Research).

Received for publication: 6 April 2011

Accepted for publication: 13 January 2012.

(C) Copyright M. Monteleone and A. Libutti, 2012

Licensee PAGEPress, Italy

Italian Journal of Agronomy 2012; 7:e6

doi:10.4081/ija.2012.e6

This article is distributed under the terms of the Creative Commons Attribution Noncommercial License (by-nc 3.0) which permits any noncommercial use, distribution, and reproduction in any medium, provided the original author(s) and source are credited. niently parameterized leaching curve prompted an attempt to extend what had been experimentally observed to a larger time and spatial scale. Therefore, different scenarios were elaborated, regarding soil salinity in relation to particular hypotheses of irrigation management and crop rotation aimed, respectively, at promoting salt leaching and minimize salt load into the soil. This has been done on the basis of historical rain-gauge series reported by the Foggia weather observatory, with reference to the years 1951-2000.

Very critical scenarios follow from the performed simulations. If only brackish groundwater is used for irrigation, the annual cultivation of a spring-summer irrigated crop without any additional leaching (apart from rain) leads to a saline buildup. This compromises agricultural soil quality. Therefore, a recommended technical choice is the cultivation of irrigated summer crops not more than once every two years. At the same time, a limited (100-200 mm) but influential fresh water applications for leaching purposes could be usefully associated.

\section{Introduction}

A sustainable agriculture in regions of insufficient or irregular precipitations depends on the proper irrigation management and the capacity to avoid that the salts brought to the soil with irrigation water accumulate into the root soil layer.

A first requirement for an effective salinity control is that soil drainage and salt leaching be apt to ensure an adequate outflow of water, together with salts, beyond the root soil layer; furthermore, the depth of groundwater should be low enough to avoid salinity problems due to capillary rise or water logging.

In order to perform leaching, appropriate water amounts should also be available; on the other side, drainage management often requires that a large amount of water with a high concentration of salts be displaced. The total volume of such water can be reduced through the improvement of irrigation systems and scheduling. Several variables affect the process:

- the seasonal precipitation regime and the annual water deficit trend of the region;

- the soil properties: texture and structure; water holding capacity and water conductivity; CEC and ESP; etc.;

- crop systems and cultivation techniques applied; particularly with reference to the seasonal amount and frequency of irrigation, crop sensitivity to drought and soil salinity, crop rotation;

- salt concentration of irrigation water and leaching strategy applied (i.e. systematic leaching application on every watering or seasonal leaching, conjunctive use of brackish and fresh water through blending or alternation, etc.);

- irrigation scheduling criteria and irrigation systems employed;

- leaching ratio as well as time of leaching application and frequency, with respect to environmental and farming conditions.

A proper agronomic management is not necessarily aimed to minimize the salts content in the root soil layer, but rather to maintain it 
within the limits compatible with a sustainable production leve (Monteleone et al., 2004). Referring to typical Mediterranean climate conditions (Capitanata geographical area, Foggia Province, Southern Italy) and assuming a recurrent and systematic irrigation use of brackish groundwater, this paper evaluates the capability of yearly rainfall, particularly in fall-winter period, to leach the salts accumulated in the soil during the previous spring-summer irrigation season. Such condition, once tested, could suggest more frequent or only occasional leaching applications, according to rain amount, salt balance and crops.

In the present work, we face the problem of estimating the amount of leaching needed to reclaim a salinized soil according to an empirically-based approach, deriving a mathematical relationship that defines practical guidelines to soil reclamation (strictly speaking, valid with respect to this type of soil only). The goals of the work can be summarized as follows:

- computation of the salt balance of the soil with respect to the induced leaching process, comparing the initial and final soil salinity conditions;

- evaluation of the water leaching effect and assessment of the residual salinity along the soil profile;

- analytical formulation of the leaching process determining the amount of leaching to be applied to gain a satisfying degree of soil reclamation (leaching curve);

- simulation scenarios obtained by applying the empirical leaching model to a long-term precipitation record related to the Capitanata geographical area, in order to give an answer to the question: is autumn-winter rain sufficient to promote salt leaching and maintain soil salt equilibrium?

\section{Material and methods}

The trial was carried out in the following steps:

- induction of a leaching effect on bare soil columns using fresh water, simulating rainfall;

- periodic recovery of drainage water and assessment of its salinity level;

- periodic determination of soil water (pore water) salinity at different depths along the soil profile;

- evaluation of soil salinity, before and after the leaching cycle, at the same depths.

\section{Experimental setup}

The trial was carried out under a rain shelter where 24 soil columns into cylindrical containers were placed. Each container was made with reinforced fiberglass and was $1.20 \mathrm{~m}$ high and $0.4 \mathrm{~m}$ in diameter, placed at about $0.20 \mathrm{~m}$ over the ground. A $0.10 \mathrm{~m}$ layer of gravel was placed in the bottom of each container, covered with a plastic net and provided with a bottom valve to collect the out-flowing drainage water into buckets.

Since the lower boundary of the soil container was exposed to atmospheric pressure, it can be concluded that free-drainage conditions were imposed. As a result, the hydraulic gradient is disrupted and differences in downward water flow and solute transport are to be found between experimental containers and field soils: solutes indeed are moving faster in field soils than in the containers. Lateral openings, distant $0.20 \mathrm{~m}$ from each other, allowed soil samples and pore-water extraction at three different heights $(0.20,0.40,0.60 \mathrm{~m})$, in order to detect their electrical conductivity. The rain shelter was used in order to prevent any dilution of the soil solutions by rainwater. The soil used for filling the containers had silt-loam texture (34\% sand; 53\% silt; $13 \%$ clay); the wilting coefficient corresponded to a relative water content of $14 \%$ in weight, while the water holding capacity (WHC) was equal to
$30 \%$ of the soil weight. The soil water content at saturation was approximately equal to $38 \%$ in weight. It can be assumed that the artificially packed soil profiles were stable because three cropping cycles had been completed inside the containers. A previous experiment conducted on the same containers spanned over three years and included two tomato growing cycles, rotated with the insertion of one durum wheat cycle. Irrigation on tomato used brackish water at various levels of electrical conductivity; as a consequence, a rapid increase in the level of soil salinity was detected during the two tomato cultivation cycles.

The experimental trial reported here was conducted during the winter of 2010 and consisted in inducing a prolonged and intense leaching cycle. This operation was carried out on a bare soil surface (i.e. soil clear from vegetation cover and usually coated with a plastic sheet except when irrigated), thus simulating a fall-winter fallow period. Fresh water was used for simulating rainfall; although fresh water ordinarily has a higher ECw value than rain water (approximately 0.05 compared to $0.02 \mathrm{dS} \mathrm{m}^{-1}$ ), this difference could be considered negligible with respect to the high salinity level (and $E C_{w}$ values) of soil water inside the containers. The leaching operations were carried out over 15 days, with a total water application of $300 \mathrm{~mm}$; soil was previously brought to water capacity with the addition of slightly brackish water $\left(E C_{w}=1 \mathrm{dS} \mathrm{m}^{-1}\right)$. The water amount daily applied to soil surface was equal to $20 \mathrm{~mm}$ (2.5 liters per container), delivered from a single dripper per container at a rate of 1 liter per hour. The rate of water supply was commensurate to the water intake rate (approximately equal to 9$10 \mathrm{~mm}$ per hour). As a consequence, a daily markedly intermittent water-flow regime was established, characterized by the succession of a wetting phase (lasting about 2.5 hours per day) and a redistribution phase, denoted by water equilibrium fluxes and deep percolation of water surplus (with a duration of about 21.5 hours per day). According to several Authors (Nielsen and Biggar, 1961; Miller et al., 1965; Nielsen et al., 1966; Oster et al., 1972; Siyal et al., 2002), intermittent leaching (as compared to continuous leaching or ponding) improves the efficiency of salt removal (more salts are removed per unit depth of water leached) because unsaturated conditions are established; this conditions allow sufficient time for solutes, between two subsequent watering, to diffuse from micro- to macro-pores and be washed away by the main convective dispersion transport stream through larger pores (Barnard et al., 2010).

The use of a single and central dripper for each container, characterized by a water supply far below the soil infiltration rate, prevented significant by-pass flows to occur through the container's lateral surface, although its diameter was relatively small. In the presence of preferential flow, however, the amount of salt leached is underestimated, while free-drainage conditions overestimate it. A sort of counterbalance effect between the two processes can be consequently assumed.

The following measurements were performed during the experiment:

daily collection of all the drainage water (corresponding exactly in volume to the daily water supply) and determination of its electrical conductivity (ECd);

- daily determination of the electrical conductivity of the soil water solution (pore water ECp) extracted at the different depths by means of a special sampler (suction cup) consisting in a porous ceramic filter, inserted into the soil at the depth of interest; a partial vacuum is created in the cup which causes water in the surrounding soil to enter it;

evaluation of the electrical conductivity of the soil saturated paste extract $\left(E C_{e}\right)$, respectively at the beginning $\left(C_{0}\right)$ and the end $(C)$ of the overall leaching process, at the three container depths.

Only the latter data will be presented and discussed in the present work. 


\section{Data processing and statistical analysis}

The 24 available containers were firstly divided into different, homogeneous groups, separated on the basis of their level of initial soil salinity $\left(C_{0}\right)$ along the soil profile as a consequence of the different irrigation salinity treatments related to the previous three-year trial. In particular, three classes were distinguished as a result of a statistical cluster analysis procedure (hierarchical clustering according to Ward's minimum variance methods). An ANOVA (analysis of variance) was then performed on the $C_{0}$ soil data with respect to the different clusters and soil columns depths; a natural logarithm transformation of the data was needed in order to homogenize variances (following the Bartlett test). A linear regression between soil salinity reduction due to leaching $\Delta C=C_{0^{-}} C$ (i.e. before and after leaching over the entire 15 days cycle) with respect to initial soil salinity $C_{0}$ was worked out. A significant influence of the soil depths was obviously expected, being the upper soil layers more intensely leached than the lower, the former transferring salts to the latter together with the percolating water. For this reason, a multi-regression analysis (GLM general linear model) was applied, statistically discriminating the three different soil depths; on this respect, an ANCOVA (analysis of covariance) was considered the proper statistical elaboration procedure.

The resulting ANCOVA slope coefficients $\left(\beta=\Delta C / C_{0}\right)$ were used to develop an empirical leaching curve, quantifying the relative amount of salts removed along the soil profile with respect to the relative amount of leaching water supplied. The latter is ordinarily expressed in terms of depth of leaching water per unit depth of soil: $D_{u} / D_{s}$; alternatively, it can also be expressed as pore water depth per unit depth of soil $D_{w} / D_{p}$, (with: $D_{p}=\theta_{\max } * D_{s}$, being $\theta_{\max }$ the maximum soil volumetric water fraction). The use of relative (instead of absolute) terms allows to derive a general and independent leaching curve, whose shape therefore is (or should be) only affected by the soil characteristics.

Jury et al. (1979), Hoffman (1980), as well as many others (e.g. Siyal et al., 2002), proposed the following empirical hyperbolic relationship:

$$
\left(C / C_{0}\right) *\left(D_{w} / D_{s}\right)=k
$$

Where $C / C_{0}$ is the fraction of the initial salt concentration remaining in the soil profile after application of the amount of water per unit depth of soil $D_{u} / D_{s}$. This equation can be significantly improved by taking the salt concentration of the applied water $\left(C_{w}\right)$ into account, the latter considered as an asymptotic equilibrium condition. The following equation was thus proposed:

$$
\left[\left(C-C_{w}\right) /\left(C_{0}-C_{w}\right)\right]^{*}\left(D_{w} / D_{s}\right)=k
$$

In an ideal porous matrix system (i.e. without pore bypass, solution of precipitated salts, salt precipitation, plant uptaking, salt diffusion constraints or hydrodynamic dispersion), the salt concentration of soil water passing through a given section in the soil profile will decrease to the concentration of the supplied water when the volume applied equals the pore space of the soil volume to be leached (Rohades and Loveday, 1990; Syial et al., 2002). However, soils seldom behave in this ideal way. An equilibrium condition is reached at the end of a very prolonged leaching process, when high volumes of applied water totally substitute the soil pore water (conditions of complete reclamation); therefore, this equilibrium value should be equal to $C_{w}$, the salinity of the irrigation water.

Another empirical hyperbolic relationship, initially suggested by Reeve (1957), is frequently used in order to reach a better fitting of the experimental data; the equation is based on two empirical coefficients ( $a$ and $b$ ) instead of one $(k)$ :

$$
\left(C-C_{w}\right) /\left(C_{0}-C_{w}\right)=a+b\left(D_{s} / D_{w}\right)
$$

According to Barnard (2010), an exponential type of function is also very suitable to describe the relationship of salt removal by leaching as a function of drainage water. A re-parameterized version of this exponential function is applied in this paper:

$$
C / C_{0}=F e q .+\left(1-F_{e q .}\right) * \exp \left[-\varepsilon /\left(1-F_{e q .}\right) *\left(D_{w} / D_{s}\right)\right]
$$

The advantage of this formulation lies in the particular meaning assigned to its parameters: $F_{e q}$, is the equilibrium (or asymptotic) salinity fraction value, while $\varepsilon$ is the initial leaching effectiveness (the water leaching capability just at the beginning of the process), geometrically corresponding to the initial slope of the curve.

Such empirical model of soil salinity remediation by leaching, properly parameterized according to the experimental data, was then applied to a long-term daily precipitation record (1951-2000) of Foggia weather station, in order to produce simulation scenarios quantifying the fraction of salts remaining in the soil, every year, as a consequence of fall-winter rains. Precipitations were computed as moving average of rainfalls over a time window of 31 days. With respect to these precipitation averages, a climatic potential leaching period was identified; it corresponds to the wet annual lapse of time during which precipitations exceed the mean rain annual value. In the arid part of the year, irrigation water is supposed to bring salts into the soil. Each yearly amount of effective leaching rainfall (ELR) is the sum of the daily effective rainfall values during the potential leaching period of the year. With respect to the 1951-2000 stretch of time, daily ELR values were determined adopting an approximate empirical criterion (Dastane, 1978) according to the following algorithm: if the day before that considered no precipitation occurred, $E L R$ is determined by subtracting the amount of $5 \mathrm{~mm}$ from the rain value of the considered day (negative values are zeroed); otherwise, if in the previous day at least $5 \mathrm{~mm}$ of rain were recorded, the $E L R$ value is equal to the actual precipitation value. Rain values less than $5 \mathrm{~mm}$ are therefore considered ineffective in promoting leaching. Since the soil is left bare, all the infiltrated water should be considered effective for leaching, because no crop transpiration consumption is supposed to occur.

As a final step, the annual $E L R$ values can be applied to the properly parameterized leaching curve equation in order to derive $C / C_{0}$, the salt fraction left in the soil along its profile at each reference depth. Considering the long-term period 1951-2000, a relative salt probability chart can be obtained; it shows the cumulative frequency distribution of residual soil salinity at the three soil depths. In this simulation, each year is considered independent from the others and randomly sorted from the 50 -year period.

Another type of simulation is also feasible; still keeping as a reference the period 1951-2000, in Foggia, it is possible to track the estimated time-course of soil salinity, at the three depths, as a result of the exact annual rainfall sequence occurring during the potential leaching period. The time course of the relative amount of soil salinity (at the three soil depths) is thus estimated applying the same parameterized salinity model. For both simulations, is assumed that each year, at each soil layer, an additional salt load corresponding to a soil $E C_{e}$ increase of $5 \mathrm{dS} \mathrm{m}^{-1}$ will be applied as a result of irrigation with brackish water on a spring-summer crop.

A third type of simulation can be proposed. A more balanced management criterion, committed to greater caution in the soil management, is based on the adoption of crop rotations inserting irrigated crops in the summer cycle only once every two years, to restrict the supply of water heavily laden with salt, thus allowing the occurrence of two successive leaching seasons. It is quite clear that, in these circumstances, it will be possible to cultivate a cereal crop (mostly durum wheat in Capitanata) during one of the two consecutive autumn-winter periods. Considering that the winter cereal is usually a rainfed crop, no further burden to soil salinity is caused. It should be considered, though, that 
not all the rainfall available in the leaching season will be used for this purpose, because a substantial part of it or even the whole must necessarily sustain wheat ET consumption. According to a prudential principle (the worst case considered), it can be assumed that no rainfall is available for leaching during the wheat growing cycle; this happens, as mentioned, in alternate years.

The simulations are run under three different conditions: 0, 100 and $200 \mathrm{~mm}$ of fresh water are respectively available to promote leaching in the fall-winter period. Each year, a fixed amount of water needs to be used to bring the water content of the soil to field capacity; the supply of $50 \%$ of the crop maximum available water is assumed, exactly at the beginning of the leaching period. This water amount is subtracted from that available to leaching.

\section{Results}

\section{Soil columns clustering}

Three relatively homogeneous containers classes of soil columns were distinguished as a result of the statistical Ward cluster procedure: Low $(L)$, Medium $(M)$ and High $(H)$ salinity soil columns were identified. Each cluster was composed by 8 containers (24 in total). The analysis of variance (ANOVA) performed on the $I n$-transformed $E C_{e}$ data, with respect to the three depths of the soil columns, showed a significant effect of clusters, but no significant effect of soil depth and also of the interaction between the two previous factors. This points out remarkable differences in the average soil salinity with respect to the three clusters (as expected), but a similar behavior in the relative trend of salinity along the soil profile. On average (Table 1), soil columns with high conductivity $(H)$ showed values equal to 20.21 , those with intermediate conductivity $(M) 11.24$ and finally those with low conductivity $(L) 5.46 \mathrm{dS} \mathrm{m}^{-1}$, the three groups being significantly different $(\mathrm{P}<0.001)$.

\section{Leaching model and leaching curve}

The applied ANCOVA model fitted separate regression lines with respect to the three soil depths. In a first model application, the average intercept value resulted not significantly different from zero; not statistically significant were also the effects of soil depths on the intercept value. Therefore, the regression lines were forced to pass through the axes origin (Table 2; Figure 1). As expected, the slope coefficients related to the three depths were significantly different from each other and gradually decreasing with soil depth; this indicates that, at equal initial salinity, the decrease in salinity induced by leaching is progressively less the greater the soil depth considered. Figure 1 shows a graphical representation of the resulting ANCOVA model; it shows a high statistical significance $(\mathrm{P}<0.001)$, a high $\mathrm{R}^{2}(0,98)$ and absence of distortion (since the regression line between observed and predicted values was not statistically different from the line 1:1).

The ANCOVA slope coefficients $\left(\beta=\Delta C / C_{0}\right)$ correspond to the relative decrease in salinity with respect to initial soil salinity, i.e. the salt fraction removed as a result of leaching at each specified soil depth.

Table 1. Average soil salinity values (ln-transformed and re-transformed to the original scale) with respect to three relatively homogeneous groups identified with the application of a cluster analysis procedure. Averages of High, Medium and Low groups are statistically different according to the ANOVA and Fischer test $(\mathbf{P}<\mathbf{0 . 0 0 1})$.

\begin{tabular}{lccc}
$\begin{array}{l}\text { Soil column } \\
\text { salinity }\end{array}$ & $\begin{array}{c}\text { Average } \\
\text { (In-transformed) }\end{array}$ & Std error & $\begin{array}{c}\text { Average } \\
\left(d S m^{-1}\right)\end{array}$ \\
High & 3.01 & 0.04 & 20.21 \\
Medium & 2.42 & 0.04 & 11.24 \\
\hline Low & 1.70 & 0.04 & 5.46 \\
\hline
\end{tabular}

Table 2. Results of the ANCOVA model applied to the soil salinity reductions due to leaching $(\Delta C$, before and after leaching, respectively) as a function of the initial soil salinity $C_{0}$ (considered as a regressor). Salinity is expressed as $E C_{e}$ (electrical con-

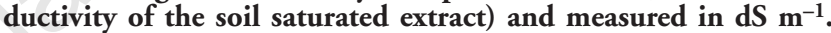
The slope values are expressed in a relative term (no unit). Considering that the intercept value was not statistically significant, it was zeroed.

\begin{tabular}{lcccc} 
& Estimate & Std error & $\mathrm{t}$ Ratio & Prob $>|\mathrm{t}|$ \\
Intercept & zeroed & - & - & - \\
slope & 0.5929 & 0.0098 & 60.36 & $<0.0001$ \\
Depth $[0.20 \mathrm{~m}]$ & +0.1120 & 0.0141 & 7.93 & $<0.0001$ \\
\hline Depth $[0.40 \mathrm{~m}]$ & +0.0028 & 0.0143 & 0.19 & 0.8470 \\
Depth $[0.60 \mathrm{~m}]$ & -0.1147 & 0.0133 & 8.64 & $<0.0001$ \\
\hline
\end{tabular}
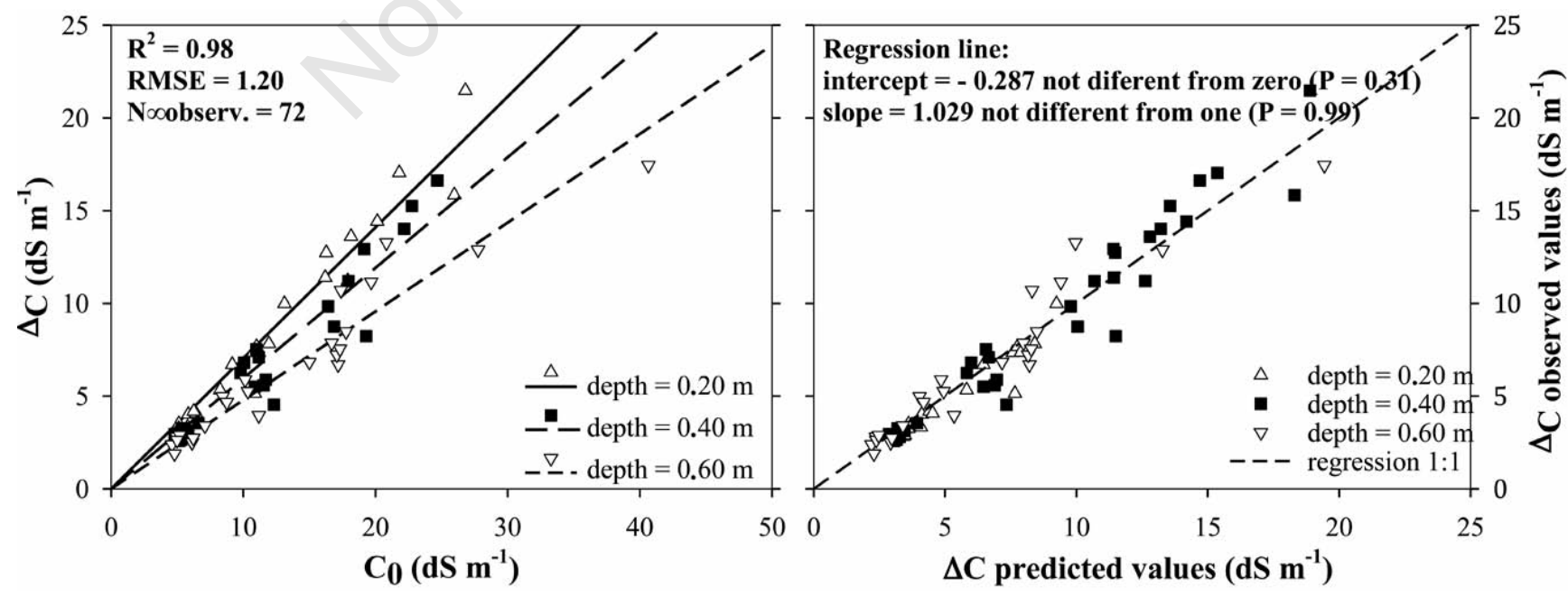

Figure 1. Left: graphical representation of the ANCOVA model carried out on soil salinity reductions $(\Delta \mathrm{C})$ due to leaching with respect to initial soil salinity values $\left(C_{0}\right)$ at the three considered soil depths $(0.20,0.40$ and $0.60 \mathrm{~m}$, respectively). Right: the good agreement between model predicted and observed values is shown. 
Three different $\beta$ values are determined, one for each soil depth. According to the $\beta$ values reported in Table 3 , it can be stated that the decrease in salinity due to leaching in the soil columns was approximately: $70 \%$ with reference to the $0-0.20 \mathrm{~m}$ soil layer, $60 \%$ in the 0.20 $0.40 \mathrm{~m}$ layer and about $48 \%$ in the layer $0.40-0.60 \mathrm{~m}$ deep. Accumulating the effects of leaching along the soil profile, it is possible to estimate a decrease of salinity respectively equal to about $70 \%$ for the layer $0-0.20$, $65 \%$ for the layer $0-0.40$, and $59 \%$ for the overall layer $0-0.60 \mathrm{~m}$. These percentages constitute the fraction of salts removed from the soil profile; the complementary value represents the fraction of salt left in the soil despite the leaching intervention (Table 3 ).

Employing the exponential equation (4), a non-linear fitting procedure was applied to the experimentally determined cumulative salinity fractions; this allowed to derive the curve presented in Figure 2. The resulting equation's parameters, together with their standard errors and confidential intervals, are reported in Table $4 ; \varepsilon$ (initial leaching effectiveness) is equal to 2.543 and $F_{e q}$ (soil salinity equilibrium fraction) to 0.295 .

\section{Effective leaching rainfall and simulation scenarios}

The long-term (1951-2000) time course of the precipitations in Foggia (calculated as moving average over a time window of 31 days) allowed to determine the climatic potential leaching period spanning from the beginning of September to the end of April (data not shown).

Considering the first simulation approach, applied to the period 1951-2000, the annual $E L R$ values (effective leaching rainfall occurring in the potential leaching period) are normally distributed with an average of approximately $200 \mathrm{~mm}$ per year and a standard error of about

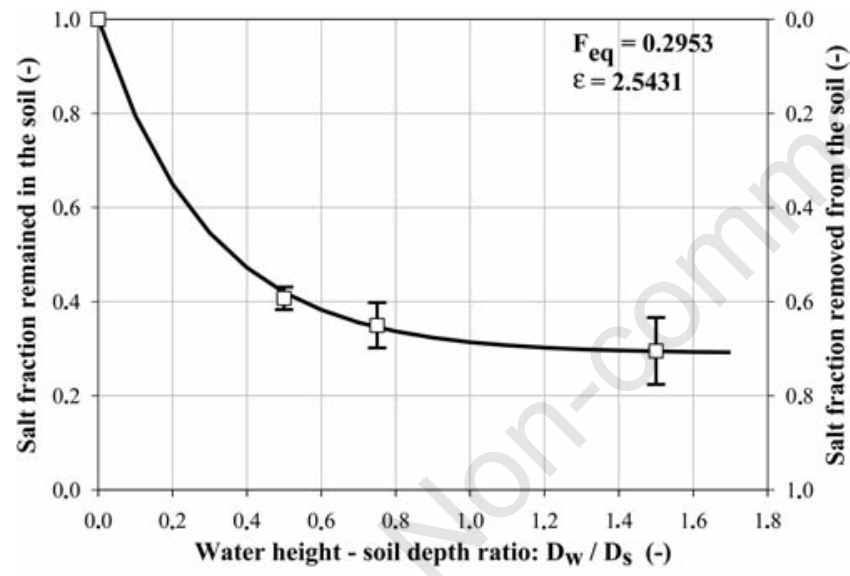

Figure 2. Leaching curve quantifying the relative amount of salts remaining in (or removed from) the soil by leaching with respect to the relative amount of leaching water supplied $\left(D_{w} / D_{s}\right)$. A negative exponential equation was fitted to the data according to the indicated parameter's values. $F_{e q}$ is the asymptotic equilibrium fraction, while $\varepsilon$ is the initial leaching effectiveness. The RMSE (Root Mean Square Error) of the model is equal to 0.073 .
$10.5 \mathrm{~mm}$ (Figure $3 \mathrm{~A})$. The resulting salt probability charts with respect to the three soil depths considered are shown in Figure 3 B,C,D. The frequency distribution of the salt fraction remained in the soil after leaching is greatly affected by the amount of the available leaching water; the latter is determined by the sum of the effective rainfall during the potential leaching period and, eventually, by a certain amount of fresh water, assumed equal to 0,100 and $200 \mathrm{~mm}$ respectively in the three lower graphs of Figure 3. Considering no supplemental water availability (Figure 3B), in $50 \%$ of cases (between the $25^{\text {th }}$ and the $75^{\text {th }}$ percentile), from 32 to $42 \%$ of the salts remain in the soil layer down to $0.20 \mathrm{~m}$ at the end of the leaching fall-winter period; from 43 to $60 \%$ with reference to the layer down to $0.40 \mathrm{~m}$; from 53 to $70 \%$ in the layer down to $0.60 \mathrm{~m}$. The availability of at least $100 \mathrm{~mm}$ of fresh water (Figure $3 \mathrm{C}$ )

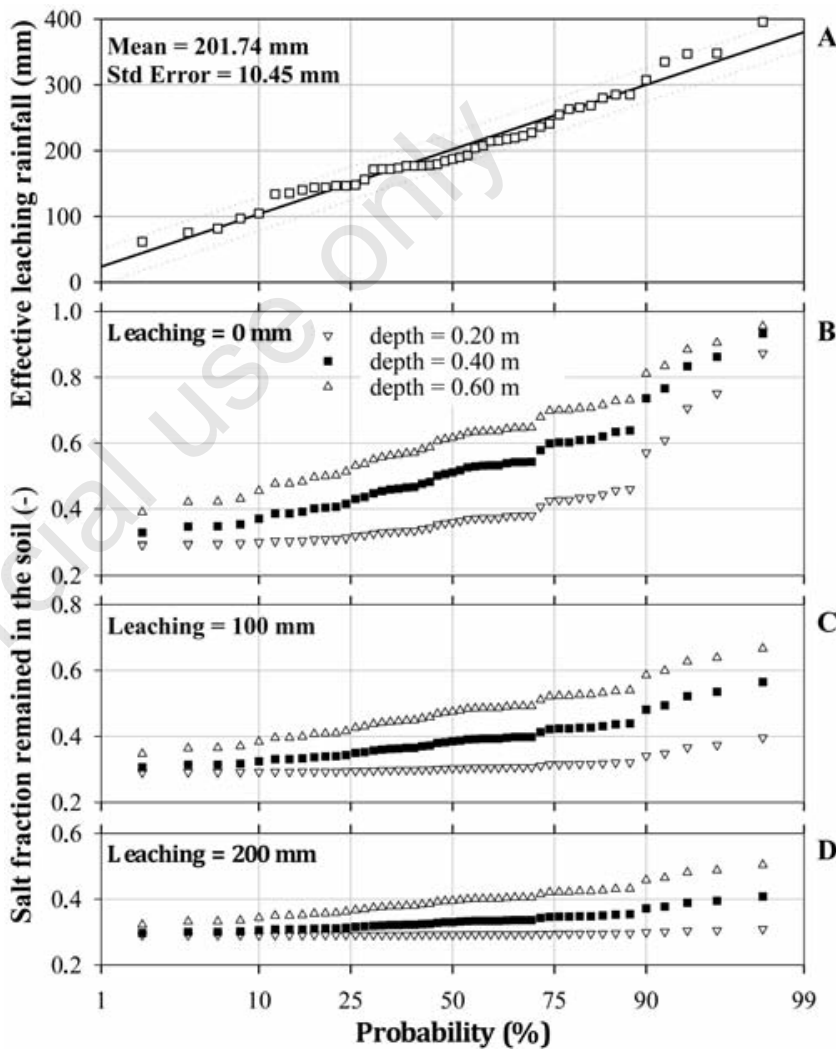

Figure 3. Effective leaching rainfall probability distribution (A) and salt probability charts $(\mathrm{B}, \mathrm{C}$ and $\mathrm{D})$. The frequency distribution of the salt fraction remaining in the soil after leaching is greatly affected by the amount of leaching water; the latter is determined by the sum of the effective leaching rainfall during the potential leaching period and the fresh water supply, equal to 0 , 100 and $200 \mathrm{~mm}$ in charts $\mathrm{B}, \mathrm{C}, \mathrm{D}$, respectively (the same also applies to Figures 4 and 5).

Table 3. Effect of leaching. Fractions of salts removed at the three soil depths and cumulative fraction of salts progressively removed along the soil profile (or correspondingly remained), together with their standard errors.

\begin{tabular}{|c|c|c|c|c|c|c|}
\hline $\begin{array}{l}\text { Soil depth } \\
(\mathrm{cm})\end{array}$ & $\begin{array}{c}\text { Fractions of } \\
\text { salts removed } \\
\text { ( } \beta \text { coefficients) }\end{array}$ & Std error & $\begin{array}{l}\text { Soil } \\
\text { profile } \\
\text { (cm) }\end{array}$ & $\begin{array}{l}\text { Cumulative fractions } \\
\text { of salts removed }\end{array}$ & $\begin{array}{l}\text { Cumulative fractions } \\
\text { of salts remained }\end{array}$ & $\begin{array}{l}\text { Cumulative } \\
\text { std error }\end{array}$ \\
\hline 20 & 0.7049 & 0.0239 & $0-20$ & 0.7049 & 0.2951 & 0.0239 \\
\hline 40 & 0.5957 & 0.0241 & $0-40$ & 0.6503 & 0.3497 & 0.0480 \\
\hline 60 & 0.4782 & 0.0231 & $0-60$ & 0.5929 & 0.4071 & 0.0711 \\
\hline
\end{tabular}


significantly improves the soil salt conditions, decreasing and narrowing the observed residual salt fractions; with respect to the same percentile range, from 30 to $31 \%$ of the salts remain in the soil layer down to $0.20 \mathrm{~m}$; from 35 to $42 \%$ with reference to the layer down to $0.40 \mathrm{~m}$; from 42 to $52 \%$ in the layer down to $0.60 \mathrm{~m}$. Finally, if $200 \mathrm{~mm}$ of fresh water are available (a surplus exactly corresponding to the average amount of seasonal effective leaching rainwater), salt control can be very strict (Figure 3D): $29 \%$ of the salts remain in the soil layer down to $0.20 \mathrm{~m}$; from 32 to $35 \%$ in the layer down to $0.40 \mathrm{~m}$; from 37 to $42 \%$ in the layer down to $0.60 \mathrm{~m}$.

According to the second simulation approach, the time-course of soil salinity in the 1951- 2000 period was estimated considering that every year a spring-summer crop irrigated with brackish water is cultivated, thus determining a regular and strong accumulation of salts along the soil profile. Figure 4 shows the results of this simulation; the amount of annual $E L R$ is reproduced at the top of the figure (Figure $4 \mathrm{~A}$ ), by means of histograms. Figure 4 B,C,D are respectively related to 0,100 and $200 \mathrm{~mm}$ of fresh additional water supply. Without additional leach-

Table 4. Estimates of the parameter values in the exponential equation employed in the non-linear fitting procedure to interpret the cumulative fraction of salts remained along the soil profile after leaching as a function of the relative leaching depth $\left(D_{w} / D_{s}\right)$.

\begin{tabular}{lcccc} 
Parameter & Estimate & $\begin{array}{c}\text { Approx. } \\
\text { std error }\end{array}$ & $\begin{array}{c}\text { Lower } \\
\text { conf. limit }\end{array}$ & $\begin{array}{c}\text { Upper } \\
\text { conf. limit }\end{array}$ \\
$\mathrm{F}_{\text {eq }}$ & 0.2953 & 0.0152 & 0.2634 & 0.3249 \\
$\varepsilon$ & 2.5431 & 0.2118 & 2.1839 & 3.0907 \\
\hline
\end{tabular}

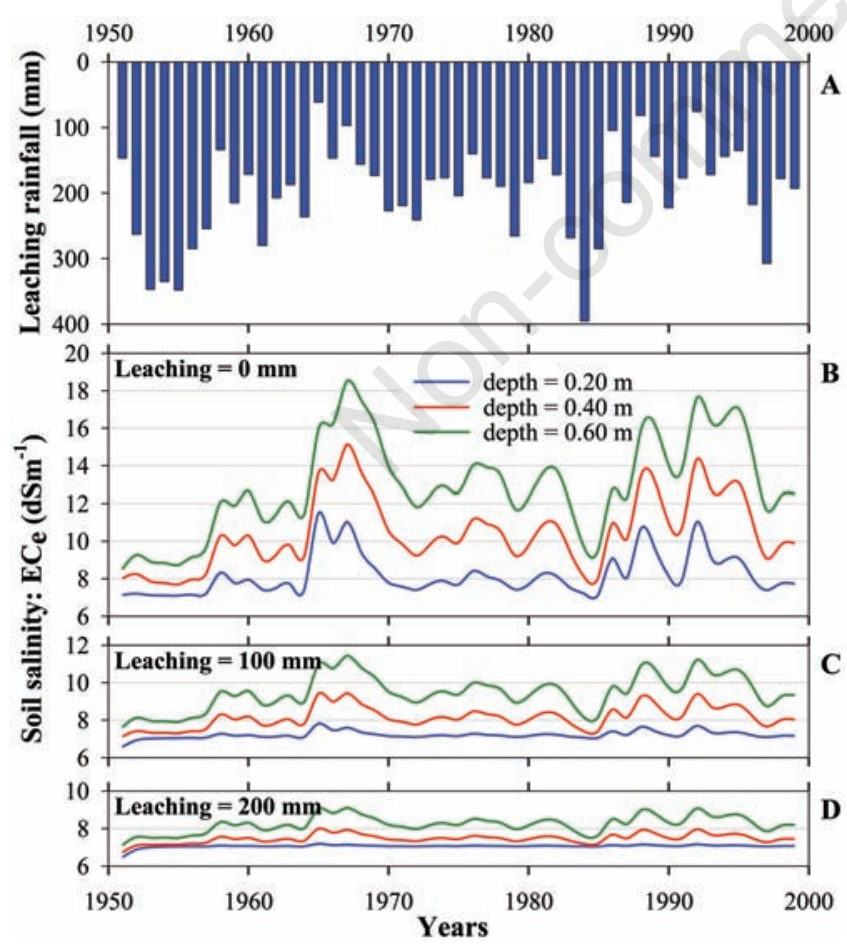

Figure 4. Simulation of the time course of soil salinity at the three considered soil depths $(0.20,0.40$ and $0.60 \mathrm{~m}) . E C_{e}$ values result from the sum of the salts remained in the soil after the previous fall-winter period and the salts added to the soil due to brackish irrigation water during the following spring-summer period. ing (apart from rainwater) the soil conditions appear clearly jeopardized; particularly in the course of protracted drought periods, salt concentrations in the soil (particularly considering the soil lower layers) reached unacceptable levels.

The third simulation is similar to the second but this time brackish irrigation water is not applied every year because irrigated crops in the summer cycle are cultivated only once every two years. Figure 5 shows the results of the simulated soil conductivity. Figure 5B shows the condition in which there is no additional leaching intervention (apart from rainwater), while on Figure $5 \mathrm{C}$ and $\mathrm{D}$ a leaching contribution of fresh water (respectively of 100 and $200 \mathrm{~mm}$ ) is considered. A reduction of soil salinity can be detected (compare Figure 4 with Figure 5) when the management is based on the alternation between summer and autumn crops; this improvement, however, is quite limited without additional leaching; differently, it becomes impressive with extra irrigation interventions with leaching purpose; a significant mitigation of soil salinity is obtained, also in those years in which the effective rainfall contributions to leaching can be considered quite low. The recurrent and periodic time-course of soil salinity shown in Figure $5 \mathrm{C}$ and especially in Figure 5D is almost independent from the yearly effective leaching rainwater; the high extra-irrigation volumes indeed played a predominant effect, thus offsetting the effect of the former.

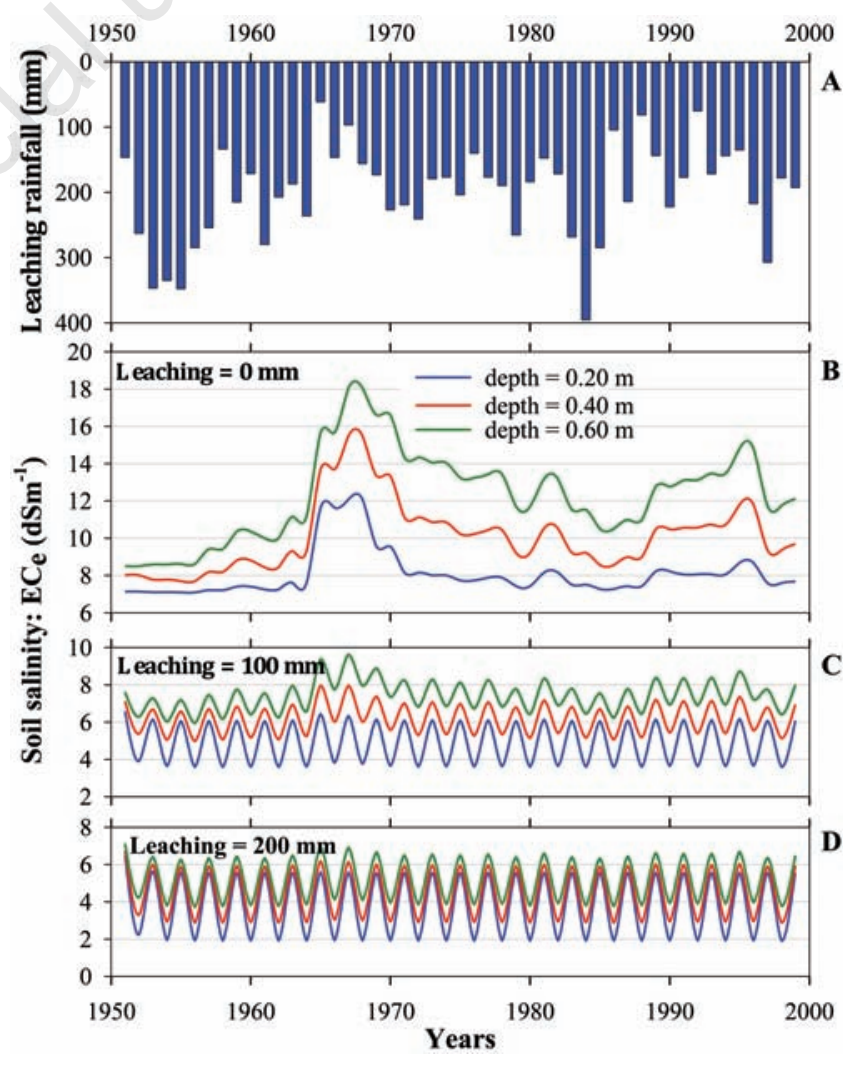

Figure 5. Simulation of the time course of soil salinity at the three considered soil depths $(0.20,0.40$ and $0.60 \mathrm{~m}$, respectively). Differently from Figure 4, in this simulation a crop rotation is applied with the insertion of an irrigated crop in the summer growing season once every two years. $E C_{e}$ values result from the sum of the salts remained in the soil after the two previous fallwinter periods and the salts added to the soil due to brackish irrigation water during the following spring-summer period. 


\section{Discussion}

The initial salinity along the soil profile, the desired salinity target after leaching, the depth to which leaching is needed and, of course, the soil characteristics are all important factors that affect the amount of water required for leaching (Abrol et al., 1988). According to the leaching curve experimentally determined, with reference to a silt-loam textured soil, basically unstructured and compact as a result of a prolonged salinizating action, the following rule of thumb can be taken: the application of a defined height of leaching fresh water reduces by $70 \%$ (i.e. reduces to $30 \%$ ) the salt content of a soil layer of equal depth. So, for example, $300 \mathrm{~mm}$ of leaching water should be used to reduce by $70 \%$ the salt content of the first $0.30 \mathrm{~m}$ of soil. If the same result is to be achieved in a layer corresponding to the first $0.50 \mathrm{~m}$ of soil, $500 \mathrm{~mm}$ of water should be used. This leaching water amount approximately corresponds to 2.1- 2.2 pore volumes of water applied. These results are in good agreement with those reported by Pazira and Sadeghzadeh (1999) and Rohades and Loveday (1990). According to the former, in general, considering the soil segments of 0.5 and 1.0 meter as the top and sub soil layers, application of one unit of leaching water per unit depth of soil (approximately two pore water) decreases the initial salt content of the respective soil layer to $78 \%$ and $60 \%$, respectively. According to the latter, about $60 \%$ and $80 \%$ total salt removal occurred with application of 2 and 4 pore volumes equivalents of leaching water, respectively. A total salt removal is both physically and practically unfeasible ( $\mathrm{Feq}$ can be theoretically reached only with unlimited leaching): a too low leaching effectiveness is observed at very high relative water depths and too much leaching water would be needed. In the presence of a very high soil salinity, large volumes of leaching water are required, which clearly highlights the exceptional significance of this technical intervention of soil reclamation. If, on the contrary, soil salinity does not reach particularly high values, remaining within the limits of a difficult but still possible cultivation practice (below threshold values around 7-8 $\mathrm{dS} \mathrm{m}^{-1}$ ), then there is the chance that autumn-winter rainfalls, especially in particularly rainy years, can result sufficient to achieve a certain amount of leaching. Further integration of water supply through additional watering may significantly increase the effectiveness of the process, in order to establish acceptable soil conditions. Very critical scenarios follow from the performed simulations; if only brackish groundwater is ordinary used for irrigation, without additional fall-winter leaching (apart from rain), a systematic annual cultivation of a spring-summer irrigated crop generates unsustainable soil salinity levels under the specific experimental conditions; even in the upper soil layer $(0-0.20 \mathrm{~m})$ the frequency of years during which the threshold of $8 \mathrm{dS} \mathrm{m}^{-1}$ is reached or exceeded corresponds to about $43 \%$; soil conditions worsen as deeper soil layers are considered, reaching very dangerous $E C_{e}$ values. These projected scenarios are not so unreliable if compared with some features of the current cultivation systems in the Capitanata area. Where summer irrigated crops are mainly cultivated, quite continuously after several years, largely using brackish groundwater with only a limited availability of fresh irrigation water, soils are undertaking a trend of increasingly serious salinity problems and farms have to face a severe constraint to agricultural productivity. The combined adoption of a biennial crop rotation and the supply of additional leaching operations with fresh water (apart from rainwater) should allow a better control of soil salinity, particularly in the shallower soil layers. Therefore, in the Mediterranean environment, the opportunity to adopt a strategy focused on the alternation between irrigated spring-summer crops and dry autumn-winter crops is highly recommended. This type of cropping system, not surprisingly, is a fundamental pillar of the traditional way of farming in the Mediterranean area. This alternation strategy can be usefully coupled to limited but effective fresh water applications for leaching purposes: 100-200 mm, if this sources are eventually available (from river or water reservoir).

\section{Conclusions}

The increasing limitation of available water resources for agriculture (both at local and global scale) raises the issue of an appropriate use of low quality water (particularly brackish or saline water) for agricultural productivity without jeopardizing the quality of soil and its productive capacity.

This work aimed at evaluating the leaching effect on a previously salinized soil, operating with fresh water simulating rainwater. This has been achieved by applying a leaching cycle on soil columns into containers with a daily water supply (exceeding the field WHC) equal to $20 \mathrm{~mm}$ for 15 consecutive days. The recording of the electrical conductivity of soil water extract $\left(E C_{e}\right)$ and porous-water $\left(E C_{p}\right)$ at different soil depths, together with the drainage water recovered at the bottom of each container, allowed to elaborate the salt balance and an appropriate quantification of the residual salinity along the soil profile.

An appropriate statistical data-processing led to the interpretation of the experimentally observed process by developing a leaching curve that predicts the fraction of salts remaining (or removed) along the soil profile according to the height of leaching water added to the soil, expressed as a fraction of the depth of the considered soil layer.

The formulation of a leaching curve, conveniently parameterized through a statistical best fitting procedure, solicited an attempt to extend what had been experimentally observed to a larger time and spatial scale. Therefore, different scenarios were elaborated, regarding soil salinity in relation to particular hypothesis of irrigation management and crop rotation, aimed at promoting salt leaching and minimize salt load into the soil. This has been done on the basis of historical rain-gauge series reported by the Foggia Weather Observatory, with reference to the years 1951-2000.

However, it should be mentioned that this procedure relies on two drastic simplifications (apart distinct differences in water flow and solute transport between experimental containers and field soils). These simplifications should be pointed out clearly in order to prevent an uncritical acceptation of the results. The first simplification concerns the assumption of the soil type and properties on which the trial took place as a general and exclusive type of the Foggia territory. In fact, it is clear that, in order to properly extend to broader areas the simulation results, it is necessary that for every different soil type or class a specific, properly configured leaching curve be worked out.

The second simplification refers to the set of rainfall data used in the scenarios elaboration. This set is limited to a single weather station (Foggia) and it does not involve the whole province of Foggia. Again, the appropriate data spatialization would require the availability of historical series covering a number of meteorological stations scattered throughout the area.

After these necessary clarifications, remains to be said that the scenarios thus generated constitute, however, a spy and a significant indication of the severity of the salinization risk, which should prompt further investigation and in depth analyses with a more articulate and detailed scenario development.

\section{References}

Abrol IP, Yadav JSP, Massoud FI, 1988. Salt-affected soils and their management. FAO Soils Bulletin N. 39. Roma, Italy.

Barnard JH, van Rensburg LD, Bennie ATP, 2010. Leaching irrigated saline sandy to sandy loam apedal soils with water of a constant salinity. Irrig. Sci. 28:191-201.

Dastane NG, 1978. Effective rainfall in irrigated agriculture. Irrigation and Drainage paper n. 25. FAO Publ., Roma, Italy. 
Hoffmann GJ, 1980. Guidelines for reclamation of salt-affected soils. pp 49-64 in Proc. Int. American Salinity Water Manage. Tech. Conf., Juarez, Mexico.

Juri WA, Jarrell WM, Devitt D, 1979. Reclamation of saline sodic soils by leaching. Soil Sci. Soc. Am. J. 43:1100-1106.

Miller RJ, Nielsen DR, Biggar JW, 1965. Chloride displacement in Panoche clay loam in relation to water movement and distribution. J. Water Resour. Res. 1:63-73.

Monteleone M, Gatta G, Giuzio L, La Rotonda P, De Caro A, 2004. Effect of leaching on salt accumulation in the soil under brackish water irrigation. pp 102-112 in Proc. Int. Workshop and Special Session on Management of poor quality waters for irrigation; 55th IEC Meeting of the International Commission on Irrigation and Drainage (ICID), Moscow, Russia.

Nielsen DR, Biggar JW, 1961. Miscible displacement in soils. Soil Sci. Soc. Am. Proc. 25:1-5.

Nielsen DR, Biggar JW, Luthin JN, 1966. Desalinization of soils under controlled unsatured flow conditions. Proc. 6th Congr. Int.
Commission on irrigation and drainage proceedings, New Delhi, India.

Oster JD, Willardson S, Hoffman GJ, 1972. Sprinkling and ponding techniques for reclaiming saline soils. Trans. ASAE 15:1115-1117.

Pazira E, Sadeghzadeh K, 1999. Soil desalinization and land reclamation in Iran. A case study: Khuzistan province. In: New technologies to combat desertification. United Nations University Press., Tokyo, Japan.

Reeve RC, 1957. The relation to salinity to irrigation and drainage requirements. Proc. 3rd Int. Congr. on irrigation and drainage, San Francisco, CA, USA, 10:175-187.

Rhoades JD, Loveday J, 1990. Salinity in irrigated agriculture. In: A. Stewart and DR Nielsen (eds.) Agronomy Monograph no. 30. American Society of Agronomy Publ., Madison, WI, USA, pp 10891142.

Siyal AA, Siyal AG, Abro ZA, Siyal AW, Memon R, Oad FC, 2002. Desalinization of aggregated saline soil: experiments on columns of spherical aggregates. Pak. J. Appl. Sci. 2:444-447. 